\title{
Detecting ECG Limb Lead-wire Interchanges Involving the Right Leg Lead-wire
}

\author{
Richard E Gregg ${ }^{1}$, E William Hancock ${ }^{2}$, Saeed Babaeizadeh ${ }^{1}$ \\ ${ }^{1}$ Philips Healthcare, Andover, USA \\ ${ }^{2}$ Stanford University, Palo Alto, USA
}

\begin{abstract}
ECG lead-wire interchanges involving the right leg $(R L)$ are not always detected. These RL lead-wire interchanges cannot be simulated in the same way as other lead-wire interchanges making database collection a necessity for algorithm development. Adult 12-lead ECGs from a single teaching hospital taken between January 2008 and July 2012 were reviewed for lead-wire interchanges by an expert electrocardiographer. Leadwire interchanges were confirmed by comparison of serial ECGs. Positive interchanges included left arm / right leg (flat lead III, $n=134$ ) and right arm / right leg (flat lead II, $n=139$ ). A RL lead-wire interchange algorithm was developed by bootstrap aggregation of decision trees with 5 -fold cross validation. Test results were summed over the 5-fold cross validation on the partitions not used for training. ECG features included maximum and minimum QRS and T-wave voltages for ECG leads I, II and III. The Haisty algorithm for RA-RL interchange was tested for comparison. Algorithm performance was quantified by sensitivity (SE), specificity $(S P)$ and estimated positive predictive value $(P P V)$ based on $S E, S P$ and realistic prevalence. For a prevalence of $0.2 \%$, performance in $S E$, SP and PPV was: Haisty, 94, 99.4, 24; tree RA-RL: 84, 99.9, 57; tree LA-RL: 87, 99.9, and 57\%. Even though $S P$ was high for all three algorithms, the estimated PPVs were modest due to the low prevalence. Conclusion: Lead-wire interchanges involving the right leg wire can be detected with good sensitivity and high specificity. The higher specificity of the tree based algorithms results in more than twice the PPV of the Haisty algorithm.
\end{abstract}

\section{Introduction}

Misconnection of the electrocardiograph lead wires to the wrong electrodes, neglecting the problem of misplaced electrodes, has been well studied with many different automated approaches to detect the common interchanges [1]. Lead-wire interchange is important to detect because the error may lead to incorrect interpretation [2]. Many different techniques were applied to automated detection, some involving signal processing, and some using common amplitude based morphology measures of the QRS and P-waves [3,4]. Assumptions which simplify the algorithm development and validation are (a) chest wires are not exchanged with limb wires because the limb wires are much longer and (b) the ground wire is not exchanged with any other lead wires. These assumptions allow simulation of lead-wire interchange using ECGs that are free from lead-wire interchange. A database can be generated by reviewing serial tracings to prove no leadwire interchange. Once the ground wire (RL) is involved however, simulation is no longer a simple matter. Positive cases must be collected using true lead-wire reversals.

While detection of limb lead wire interchange with RL is generally simple - one of the limb leads is nearly flat the frequency is rare therefore an automated algorithm must have very high specificity to maintain a reasonable positive predictive value. Hedén found the frequency of RA-RL interchange to be just $0.3 \%(31 / 11,423)$ and the LA-RL and arm-for-leg interchanges were presumably lower frequency and lumped together with "other" [3].

The aim of this study was to design and validate an algorithm for interchanges of the RL wire with the left arm and right arm lead-wires. As part of the validation, the automated algorithm presented here was compared to the Haisty algorithm for RA-RL interchange [5].

\section{Methods}

Study ECGs were a subset from a larger effort to collect examples of lead-wire interchange. In this subset, we concentrated on the RA-RL and LA-RL interchanges. ECGs were excluded because of excessive noise obscuring the $\mathrm{P}$ wave, paced rhythm, or P-wave superimposed on the previous T-wave. Arrhythmias such as atrial fibrillation, atrial flutter, supraventricular tachycardia, ectopic atrial rhythm and junctional rhythm are included however. ECGs with features consistent with potential RL lead wire interchange were compared with the patient's previous or later ECGs to confirm the interchange. The control set without RL interchanges was allowed to contain interchanges limited to the precordial leads.

The Philips DXL algorithm was used to generate a measurement matrix and a representative PQRST complex 
for each ECG. Various features consistent with flat lead II

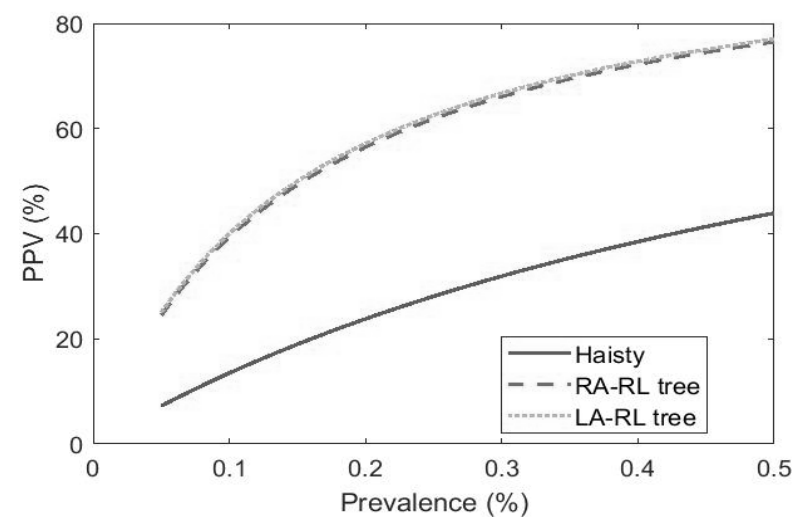

Figure 1. PPV estimated from SE, SP and prevalence of lead-wire interchange.

and flat lead III were included in the analysis. Those features included R, S and T-wave amplitudes and peakto-trough voltages of QRS and T-waves. In addition, ECGs were pre-filtered with a single pole $40 \mathrm{~Hz}$ lowpass filter as input to the Haisy algorithm.

Bootstrap aggregation of decision trees or "bagged" trees was the method for classifying ECGs as RA-RL, LA$\mathrm{RL}$ or no lead wire interchange. With the bagged tree method, a decision tree is trained for each bootstrap resampling of the training set. The final classification is determined by vote across the set of trees. K-fold cross validation with 5 folds was used for training and testing. The training was performed on 4 folds and tested on the remaining fold. This was repeated across the 5 folds to end up with test results spanning the whole set.

Algorithm performance was measured by sensitivity (SE), specificity (SP) and positive predictive value (PPV). Since the data set was selected as two separate groups, a positive and control group, PPV was estimated from SE, SP and prevalence (PREV) [6]. For comparison, the Haisty algorithm for RA-RL lead interchange was tested on the same dataset [5].

\section{Results}

Algorithm performance is summarized in One of the design outputs of the bagged tree classifier is the importance of the features. The most important features were related to flat lead II and flat lead III as expected, (a) QRS peak-to-peak voltage, leads II and III, (b) T-wave peak-to-peak voltage, leads II and III and (c) ratio of QRS peak-to-peak amplitude in lead II vs. lead III. Interestingly, QRS axis was not an important feature.

Table 1. While all three algorithms have high specificity, the estimated PPV is only moderate for the expected range of prevalence. The small difference in specificity between the Haisty and tree based RA-RL algorithms results in a doubling of PPV. The higher PPV of the tree based classifier can clearly be seen in Figure 1, PPV versus prevalence of lead-wire interchange. The prevalence of the RA-RL interchange is expected to be around $0.3 \%$ while the prevalence of the LA-RL interchange should be even lower.

The confusion matrix in Table 2 shows the exact counts used to generate the performance numbers in One of the design outputs of the bagged tree classifier is the importance of the features. The most important features were related to flat lead II and flat lead III as expected, (a) QRS peak-to-peak voltage, leads II and III, (b) T-wave peak-to-peak voltage, leads II and III and (c) ratio of QRS peak-to-peak amplitude in lead II vs. lead III. Interestingly, QRS axis was not an important feature.

Table 1 . Note that the number of false positives is just 2 for the tree based algorithms.

One of the design outputs of the bagged tree classifier is the importance of the features. The most important features were related to flat lead II and flat lead III as expected, (a) QRS peak-to-peak voltage, leads II and III, (b) T-wave peak-to-peak voltage, leads II and III and (c) ratio of QRS peak-to-peak amplitude in lead II vs. lead III. Interestingly, QRS axis was not an important feature.

Table 1. Algorithm performance

\begin{tabular}{|c|c|c|c|c|}
\hline Algorithm & $\begin{array}{l}\mathrm{SE} \\
(\%)\end{array}$ & $\begin{array}{l}\text { SP } \\
(\%)\end{array}$ & $\begin{array}{c}\text { PREV } \\
(\%)\end{array}$ & $\begin{array}{l}\text { PPV } \\
(\%)\end{array}$ \\
\hline \multirow{3}{*}{$\begin{array}{l}\text { Haisty } \\
\text { RA-RL }\end{array}$} & \multirow[t]{3}{*}{93.5} & \multirow[t]{3}{*}{99.4} & 0.3 & 32 \\
\hline & & & 0.2 & 24 \\
\hline & & & 0.1 & 14 \\
\hline \multirow{3}{*}{$\begin{array}{l}\text { Tree based } \\
\text { RA-RL }\end{array}$} & \multirow[t]{3}{*}{84.2} & \multirow[t]{3}{*}{99.9} & 0.3 & 66 \\
\hline & & & 0.2 & 57 \\
\hline & & & 0.1 & 39 \\
\hline \multirow{3}{*}{$\begin{array}{l}\text { Tree based } \\
\text { LA-RL }\end{array}$} & \multirow[t]{3}{*}{86.6} & \multirow[t]{3}{*}{99.9} & 0.3 & 67 \\
\hline & & & 0.2 & 57 \\
\hline & & & 0.1 & 40 \\
\hline
\end{tabular}

Table 2. Confusion matrix for the Haisty and tree based classifiers

\begin{tabular}{l|cc|ccc}
\hline $\begin{array}{l}\text { Ref } \backslash \\
\text { Alg }\end{array}$ & $\begin{array}{c}\text { RA-RL } \\
\text { Haisty }\end{array}$ & $\mathrm{N}$ & $\begin{array}{c}\text { RA-RL } \\
\text { tree }\end{array}$ & $\begin{array}{c}\text { LA-RL } \\
\text { tree }\end{array}$ & $\mathrm{N}$ \\
\hline RA-RL & 130 & 9 & 117 & 0 & 22 \\
LA-RL & NA & NA & 2 & 116 & 18 \\
N & 9 & 1435 & 0 & 2 & 1441 \\
\hline
\end{tabular}

\section{Discussion}

We presented the design and performance of a decision tree based algorithm for detection of lead-wire interchange between the right leg and arm electrodes. This algorithm compares favorably to the only published algorithm we 
could find for right arm and leg interchange by Haisty [5]. Our algorithm detects both arm interchanges with right leg. Although the sensitivity of our algorithm is lower for the RL-RA interchange, we feel the clinical acceptance will be higher because the PPV is much higher. When clinicians perceive a high number of false positives, often the algorithm will be turned off. The Haisty algorithm is very simple so it can be used in manual reading while our algorithm is suitable for an automated system, not manual reading.

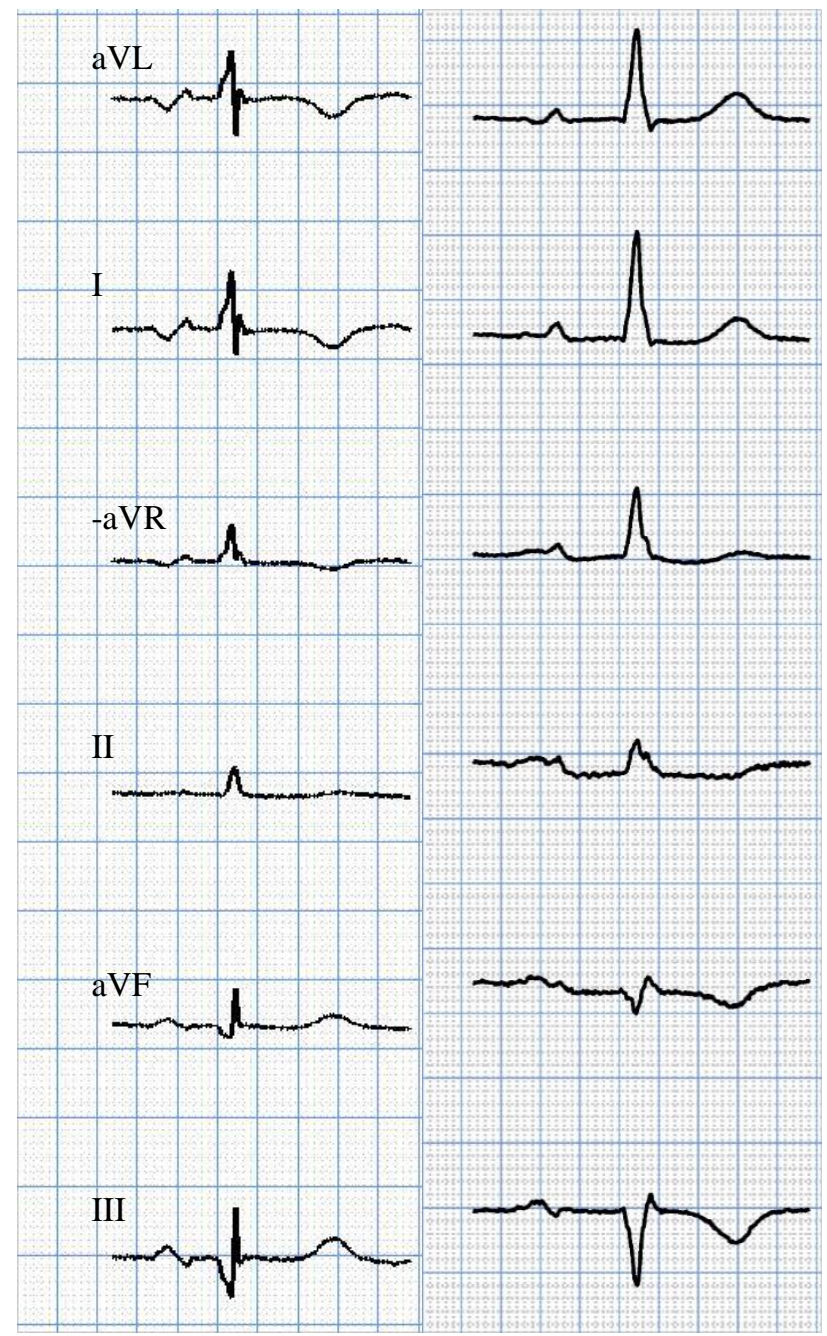

Figure 2. True positive (left) and false positive (right) RA-RL interchange. Leads are presented in Cabrera order. Lead II should be flat with a RA-RL reversal.

Figure 2 and Figure 3 show examples of lead wire interchange, both true positives and potential false positives. These examples were chosen not to display textbook examples of how the ECG morphology appears but to show the overlap of interchange and no interchange where false positives can clearly occur. The voltage difference between the legs is not truly zero, therefore the amplitude in leads II and III can approach zero but in some cases there is a significant non-zero amplitude as shown in the figures. When the frontal plane QRS and T-wave axis are near either 30 or -30 degrees, biphasic or low amplitude deflections in leads III and II can result. Those low amplitude deflections could appear to be lead-wire interchange.

Automated lead wire interchange involving the right leg wire may not have high enough performance for MasonLikar (ML) electrode placement on the torso. Batchvarov explained clearly how the voltage difference between leg

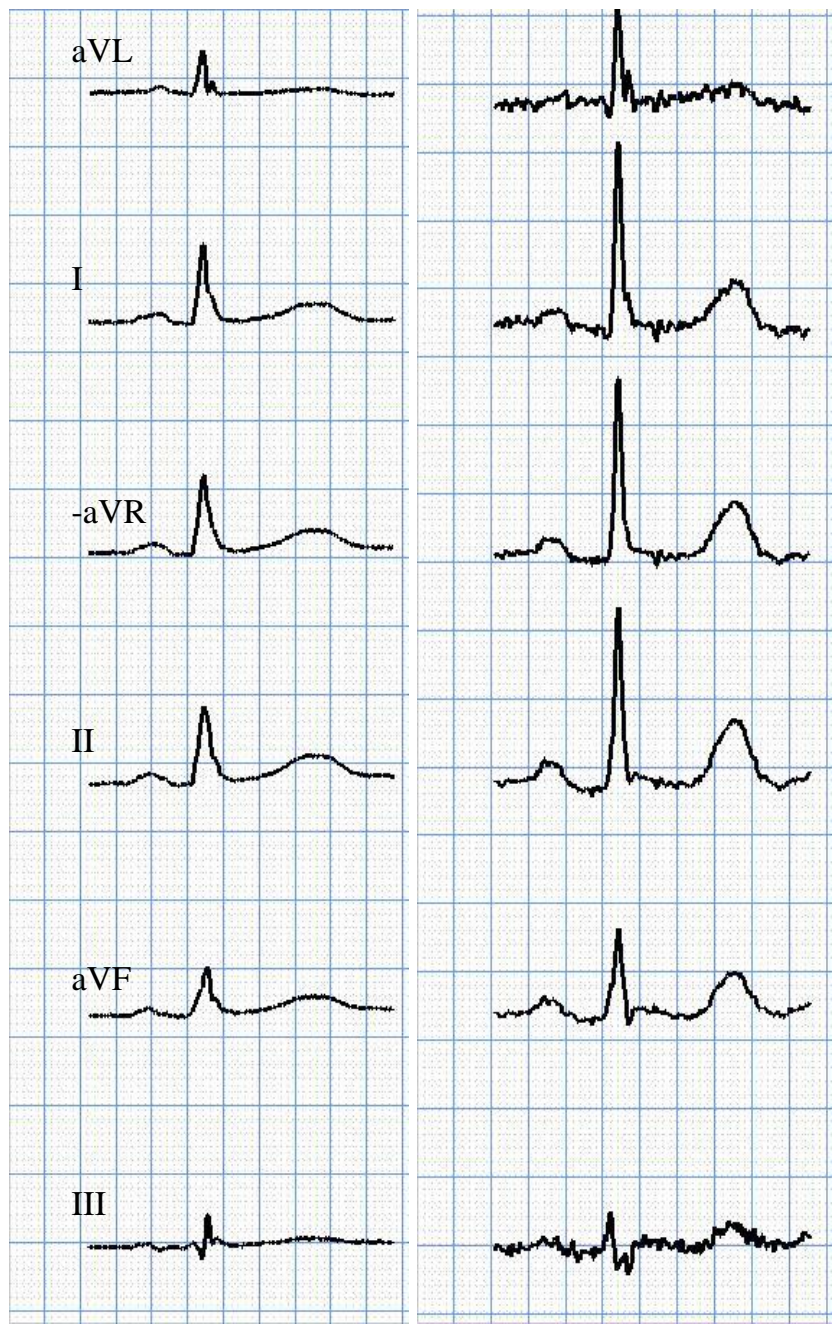

Figure 3. True positive (left) and false positive (right) LA-RL interchange. Lead III should be flat with a LA$\mathrm{RL}$ reversal.

electrodes increases as the electrodes are moved up the legs onto the torso and closer to the ribs [1]. With higher voltages between leg electrodes, amplitude thresholds must also increase to maintain high specificity for RL interchanges but higher thresholds will result in more false negatives and lower sensitivity. Leaving the thresholds as is should decrease specificity for ML electrode placement. Performance may still be acceptable for ML placement in 
some areas of the hospital where the prevalence of lead wire interchange has been shown to be higher because PPV increases with prevalence. Rudiger for instance found $4 \%$ prevalence for all lead-wire interchange in the ICU versus $0.4 \%$ in the outpatient clinic [7]. For other lead wire interchanges, ML placement may not be a problem. Han found no significant impact due to ML placement for nonRL limb lead interchange [8].

The low prevalence of this type of lead-wire interchange means that even a good specificity will result in a poor positive predictive value. To get that reasonable PPV and extraordinarily high specificity, either the control group must be very large or the design process must heavily favor low false positives over false negatives. In our case, the optimization for low prevalence involved setting the cost for a false positive to be roughly 5 times the cost of a false negative. The main problem is that there are still very few false positives and because the number is so low, we cannot say much about what signal or feature characteristics cause false positives.

\section{Conclusion}

Lead-wire interchanges involving the right leg wire can be detected by algorithm with good sensitivity and high specificity. The higher specificity of the tree classifier presented here resulted in more than twice the PPV of the Haisty algorithm. The important features were related to flat lead II and lead III as expected, both QRS and T-wave peak-to-peak amplitudes.

\section{References}

[1] Batchvarov VN, Malik M, Camm JA. Incorrect electrode cable connection during electrocardiographic recording. Europace. 2007; 9: p. 1081-1090.

[2] Guijarro-Morales A, Gil-Extremera B, Maldonado-Martín A. ECG diagnostic errors due to improper connection of the right arm and leg cables. International Journal of Cardiology. 1991; 30(2): p. 233-5.

[3] Hedén B, Ohlsson M, Holst H, Mjijman M, Rittner R, Pahlm O, et al. Detection of Frequently Overlooked Electrocardiographic Lead Reversals Using Artificial Neural Networks. Am J Cardiol. 1996; 78: p. 600-4.

[4] Han C, Gregg RE, Feild DQ, Babaeizadeh S. Automatic detection of ECG cable interchange by analyzing both morphology and interlead relations. J Electrocardiol. 2014; 47(6): p. 781-7.

[5] Haisty WK, Pahlm O, Edenbrandt L, Newman K. Recognition of electrocardiographic electrode misplacements involving the ground (right leg) electrode. Am J Cardiol. 1993; 71(16): p. 1490-5.

[6] Altman D, Machin D, Bryant T, Gardner S. Statistics with Confidence: Confidence intervals and statistical guidelines. 2nd ed. Bristol: BMJ Books; 2000.

[7] Rudiger A, Hellermann JP, Mukherjee R, Follath F, Turina J. Electrocardiographic artifacts due to electrode misplacement and their frequency in different clinical settings. Am J Emerg Med. 2007; 25(2): p. 174-8.

[8] Han C, Gregg RE, Babaeizadeh S. Automatic detection of ECG lead-wire interchange for conventional and Mason-Likar lead systems. In Computing in Cardiology; 2014: IEEE. p. 145-148.

Address for correspondence.

Richard E. Gregg

3000 Minuteman Rd, MS4201

Andover, MA 01810

rich.gregg@philips.com 